\title{
Stem Carbohydrates and Adventitious Root Formation of Cycas micronesica following Aulacaspis yasumatsui Infestation
}

\author{
Thomas E. Marler ${ }^{1}$ \\ University of Guam, CNAS, WPTRC, UOG Station, Mangilao, Guam \\ Additional index words. adventitious roots, cycad, fructose, glucose, starch, sucrose
}

\begin{abstract}
Large stem cuttings were removed from Cycas micronesica K.D. Hill trees and used for asexual propagation to more fully understand resource factors that influence adventitious root formation success. Healthy source trees had received insecticide protection from ubiquitous Aulacaspsis yasumatsui Takagi infestations and unhealthy source trees had suffered from chronic $A$. yasumatsui infestations. Nonstructural carbohydrates were quantified from stem tissues at the base of each 1-m cutting, and induction of adventitious roots was attempted using field soil as the medium. Carbohydrate concentrations were increased in healthy, protected tree cuttings above those in unhealthy, infested tree cuttings. The relative increase was greatest in the hexoses, intermediate in sucrose, and least in starch. The total nonstructural carbohydrate concentration in cuttings of infested trees was $54 \%$ of that of protected trees, and the sugar/starch quotient of infested trees exceeded that of protected trees. Asexual propagation success was $30 \%$ for the unhealthy tree cuttings and $100 \%$ for the healthy tree cuttings. These manipulative experiments confirm that chronic $A$. yasumatsui herbivory of $C$. micronesica trees reduces stem carbohydrates and decreases asexual propagation success. The results indicate that the use of large cuttings from unhealthy $C$. micronesica trees to rescue tree populations from construction sites is not a wise conservation decision in habitats where $A$. yasumatsui herbivory has been uncontrolled. Protocols for future rescue operations designed to transplant $C$. micronesica trees from construction sites may be improved by this new knowledge.
\end{abstract}

Cycas micronesica was listed as Endangered under the International Union for Conservation of Nature (IUCN) in 2006 (Marler et al., 2010) and Threatened under the United States Endangered Species Act (ESA) in 2015 (United States Fish \& Wildlife Service, 2015). The species is Guam's only native gymnosperm and the only native Cycas species within the United States. The abrupt change in conservation status was caused by the invasions of several specialist insect pests to the islands of Guam and Rota, with the primary threat developing from the 2003 invasion of A. yasumatsui (Hemiptera: Diaspididae) (Marler, 2012; Marler and Muniappan, 2006). This armored scale species feeds on the surfaces of all vegetative and reproductive organs, and the lack of biological control in Guam's insular settings caused mortality of more than $90 \%$ of the Cycas plants in urban and natural habitats (Marler and Lawrence, 2012).

The local community of biologists has responded to the need to conserve threatened

Received for publication 25 Apr. 2018. Accepted for publication 31 May 2018 .

Supported in part by US Forest Service Project No. 06-DG-11052021-206 and 10-DG-11059702-095 and USDA National Institute of Food and Agriculture McIntire-Stennis project 1005274.

${ }^{1}$ Corresponding author. E-mail: thomas.marler@ gmail.com. native plant species without complete knowledge, so nascent conservation projects have adopted an adaptive management approach. This is a common phenomenon, and much has been written about funders, policymakers, and practitioners needing to respect the vital importance of evidence-informed conservation decisions, especially when empirical information is scarce or scattered (Marler and Lindström, 2017; Pullin and Knight, 2009). The paucity of relevant local research on threatened terrestrial plant species has limited conservation successes in the Mariana Islands (Marler, 2017; Marler and Lindström, 2014). This phenomenon is not limited to the Mariana Islands with regard to cycad conservation issues, as publications on horticulture and physiology of cycads are deficient worldwide (Cascasan and Marler, 2016).

The potential for the use of large stem cuttings for rescue projects designed to transplant $C$. micronesica tree populations from military construction sites was recently evaluated (Marler and Cruz, 2017) because excavation of intact roots for traditional transplant operations was cost-prohibitive. This unprecedented attempt to initiate adventitious roots on large cycad stems resulted in $41 \%$ success in adventitious root formation under management by experienced cycad horticulturists and 100\% mortality under management by silviculturists possessing no cycad experience. The outcomes illuminated 1) the necessity of applied cycad horticultural experience for practitioners of cycad conservation and restoration and 2) that large stem cuttings may be further considered for tree restoration projects only if more research identifies constraints to improved success rates.

Asexual propagation of healthy Cycas plants is routinely accomplished by inducing root formation on small adventitious stem cuttings (Norstog and Nicholls, 1997). The relatively low success rate for large C. micronesica stem cuttings may have resulted from the unhealthy status of the stems due to chronic A. yasumatsui damage to the donor plants (Marler and Cruz, 2017). To more fully understand how Cycas plants respond to chronic A. yasumatsui infestations, Cycas revoluta Thunb. plants were experimentally infested and then tissues harvested to determine carbohydrate status (Marler and Cascasan, 2018). The results confirmed that $87 \%$ of the nonstructural carbohydrates were depleted during the 60 weeks of infestation that were required to reach $C$. revoluta plant mortality.

Several locations throughout Guam were selected in 2007 for attempts to protect in situ trees from the A. yasumatsui threat by using insecticides. The endeavor began with stem injection protocols, but the damage to the persistent, live cortex tissue of Cycas stems was too extensive to implement stem injection on a large scale or sustained basis (Fisher et al., 2009). Root drenches of imidacloprid and dinotefuran systemic insecticides were subsequently evaluated for efficacy. The dinotefuran was not effective under Guam's climate and soils, but the efficacy of imidacloprid was adequate to suppress $A$. yasumatsui pressure. This form of plant protection within the dedicated sites was continued with sustained drenches every 3-4 months.

A manipulative experiment was clearly needed to confirm the role of carbohydrates in the compromised adventitious root induction of unhealthy $C$. micronesica stem cuttings. Therefore, two of the Guam sites containing healthy trees that had been protected with imidacloprid were selected to conduct a propagation experiment that included direct carbohydrate quantification of each cutting. The objectives were to confirm the direct connection between nonstructural carbohydrate depletion in stems by A. yasumatsui herbivory and failures in asexual propagation attempts with $C$. micronesica trees.

\section{Materials and Methods}

Cycas micronesica trees with multiple leaders were selected from a southern Guam and an eastern Guam site that contained healthy trees that were protected from $A$. yasumatsui by imidacloprid (Fig. 1A) and unhealthy trees that were infested with $A$. yasumatsui (Fig. 1B). The field work was conducted in June 2013 at a time the healthy trees had received insecticide protection for 
6 years and the unhealthy trees had been infested with $A$. yasumatsui for 7 years. The southern site was positioned on volcanic soils formed in residuum derived from tuff (very fine, kaolinitic, isohyperthermic Oxic Haplustalfs), and the eastern site was positioned on coralline soils formed in slope alluvium, loess, and residuum overlying limestone (clayey-skeletal, gibbsitic, nonacid, isohyperthermic Lithic Ustorthents) (Young, 1988).

Trees with multiple branched stems were selected to ensure each donor plant retained several growing apices following removal of the subapical experimental stem cuttings. The wound on each donor plant was treated by allowing the surface to air-dry for several hours and then covering with commercial pruning sealant. Each cutting was $100 \mathrm{~cm}$ in length, and the basal $1 \mathrm{~cm}$ of the entire stem was removed for carbohydrate analysis. The tissue was immediately placed in an ice chest and then transferred the same day for longterm storage at $-20{ }^{\circ} \mathrm{C}$ until resources were available to conduct the carbohydrate analyses (described in the following paragraphs).

The propagation protocols followed those of Marler and Cruz (2017). All leaves and debris were removed from the apex. The base of each stem cutting was treated in sequence with $10 \%$ bleach solution, tetrachloroisophthalonitrile fungicide, and commercial $0.3 \%$ indole-3-butyric acid product. The open basal surface of each cutting was allowed to air-dry for several hours and then covered with a commercial pruning wound sealant. The cuttings were inserted vertically into $15-\mathrm{cm}$ holes near the donor tree to mimic the natural cloning of detached stems that occurs during Guam's recurring tropical cyclones (Marler and Lawrence, 2013). Two distinct seasons include a dry season roughly from December to June when the mean monthly rainfall is $11.6 \mathrm{~cm}$, and a rainy season roughly from July to November when the mean monthly rainfall is $29.8 \mathrm{~cm}$ (www.wunderground. com). Therefore, late June was selected for these procedures to ensure the cuttings were provided with adequate rainfall throughout the entire rainy season of 2013. The cuttings were inspected bimonthly and provided imidacloprid drench on the same schedule with the donor trees. Inspections were ended in Aug. 2014 when propagation success of each cutting was determined. All successful cuttings contained numerous healthy leaves within several age categories. Cuttings with no leaves and with decomposing stems were evaluated as dead.

All frozen tissues were lyophilized and then milled to pass through a $1-\mathrm{mm}$ screen. Methods for carbohydrate quantification followed Marler and Dongol (2016). Soluble sugar extraction was conducted using hotwater extraction with acetonitrile $\left(80{ }^{\circ} \mathrm{C}\right.$; Schloter et al., 2005). The concentrations of four free sugars (the hexoses fructose and glucose, and the disaccharides sucrose and maltose) were determined using HPLC-RI (Thermo Scientific RI-150, AS3000 autosampler, P2000 pump; Thermo Scientific, Waltham, MA). Starch was quantified using the anthrone colorimetric method following removal of sugars by ethanol and then hydrolysis of starch to glucose using sulfuric acid at $95{ }^{\circ} \mathrm{C}$ (Clegg, 1956).

We also calculated the total free sugar content by adding the concentration of all four sugars and the total nonstructural carbohydrate content by adding the concentration of all five carbohydrates. The relationship of sugars to starch was calculated as total free sugar content divided by starch. The data were subjected to a $2 \times 2$ factorial analysis of variance with two locations as one factor and two levels of armored scale infestation as the second factor with 10 replications, using Proc MIX function in SAS (SAS Institute, Cary, NC). Hereinafter, the unprotected infested trees will be labeled unhealthy and the protected uninfested trees will be labeled healthy. The propagation success rate data were not subjected to statistical analysis.

\section{Results and Discussion}

The $C$. micronesica stem carbohydrates responded similarly to imidacloprid protection from A. yasumatsui herbivory for each of the two sites, so the site $\times$ infestation level interaction was not significant for any of the chemical traits. Moreover, the carbohydrate status of the stems was similar for the two sites, so the main effect of site was also not significant for any of the chemical traits. In contrast, the main effect of armored scale infestation level exhibited significance for all carbohydrate response traits, except for maltose
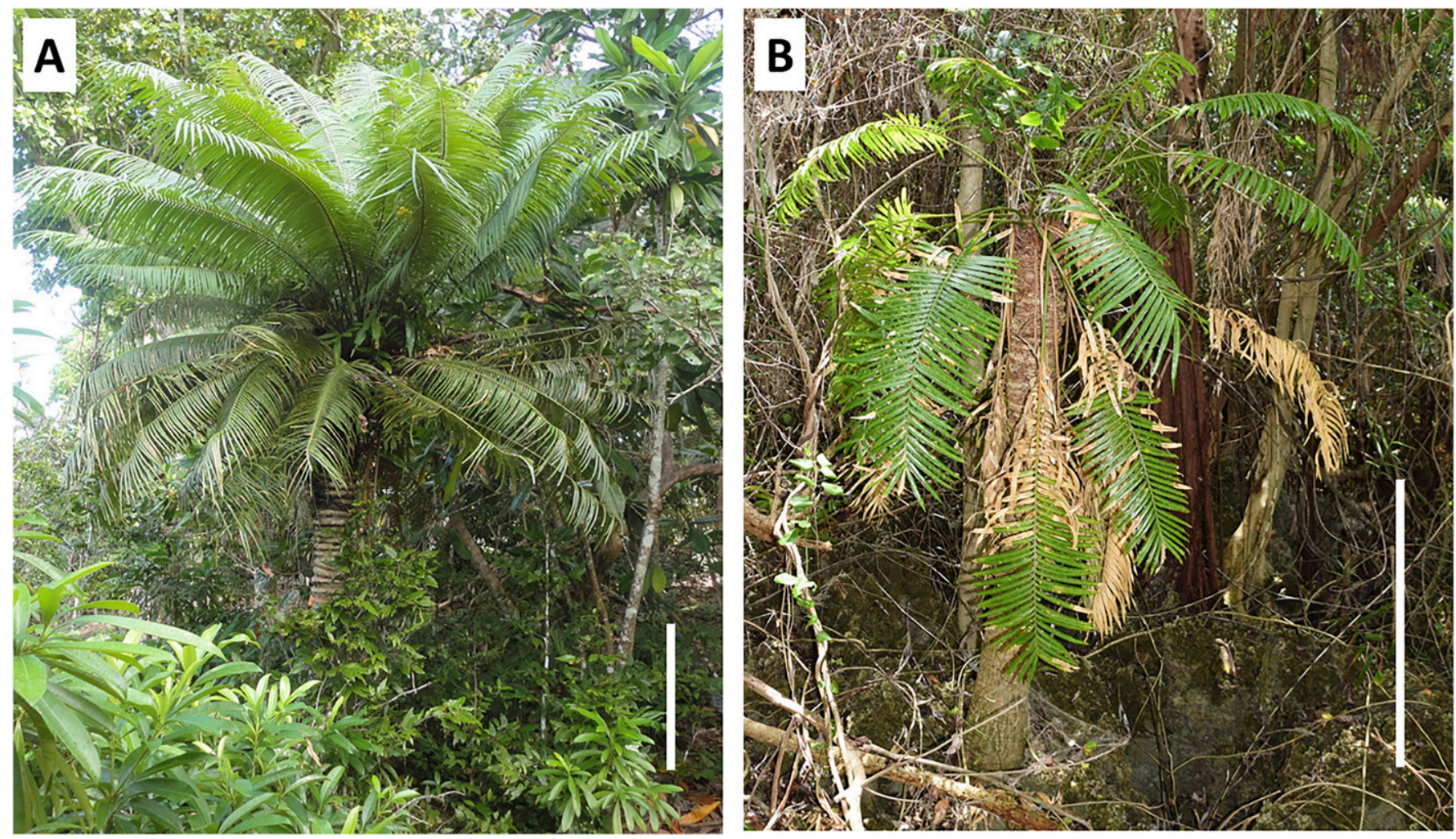

Fig. 1. Characteristic Cycas micronesica trees on Guam in June 2013. (A) Healthy tree following long-term imidacloprid protection from Aulacaspis yasumatsui herbivory revealing numerous robust leaves. (B) Unhealthy tree provided no insecticide protection revealing few small leaves. White bars are $100 \mathrm{~cm}$. 
concentration (Table 1). Fructose concentration of stems from healthy trees was 2.4-fold greater than that of unhealthy trees. Stem glucose concentration of healthy trees was 4.4-fold greater than that of unhealthy trees. Stem sucrose concentration was more than 5 times greater than that of fructose and more than 9 times greater than that of glucose. Stem sucrose of healthy trees was 2.1-fold above that of unhealthy trees. Maltose concentration was minimal for all plants and was not influenced by imidacloprid protection. Starch concentration exceeded that of each of the free sugars, and the stem starch concentration of healthy trees was 1.6-fold greater than that of unhealthy trees.

Total free sugar concentration of the stems of unhealthy $C$. micronesica trees was $44 \%$ of that of healthy trees (Table 1). Similarly, total nonstructural carbohydrate concentration of the stems of unhealthy trees was $54 \%$ of that of healthy trees. Although imidacloprid protection increased the concentrations of the free sugars and starch above the concentrations of unprotected trees, the relative increase in sugars exceeded that of starch. Therefore, the sugar/starch quotient of unhealthy trees exceeded that of healthy trees.

All of the cuttings from healthy trees that had been protected from $A$. yasumatsui infestations were successfully propagated and vigorously growing in Aug. 2014. By contrast, only $20 \%$ of the cuttings from unhealthy trees in the southern site and $40 \%$ of these cuttings in the eastern site were successful. Cycad stems primarily comprise parenchyma, so the unhealthy stems that did not possess the ability to produce adventitious roots were partially decomposed in Aug. 2014 and added to the standing litter pool. All of the 40 donor trees revealed no change in health status between June 2013 and Aug. 2014 , verifying that excision of the subapical cuttings did not influence the health of the trees.

The relationships among sugars and starch were similar for this field study where insecticides enabled $C$. micronesica trees to recover from $A$. yasumatsui herbivory and our former nursery study where $C$. revoluta plants were forced to respond to experimentally initiated $A$. yasumatsui herbivory until plant death occurred (Marler and Cascasan,
2018). For example, stem sugar/starch of the healthy plants was 1.21 in this $C$. micronesica study and 1.29 in the $C$. revoluta study, and this metric greatly increased in the unhealthy, infested plants in both studies. Stem starch was clearly metabolized and expended in excess of stem sugars as plants of both species responded to chronic A. yasumatsui herbivory.

Locally relevant manipulative studies are useful for generating empirical data to inform ongoing adaptive management decisions by creating a more complete picture on which to base conservation decisions (Cooke et al., 2018; Marler, 2017; Marler and Lindström, 2014, 2017; Zedler, 2017). The transplantation plans for the rescue of threatened tree species from Guam's ongoing construction sites generally do not require the rescue of the entire tree population within the construction footprint. For these projects, the gestalt appearance of each tree has been used to determine which specimens were selected for rescue. The results herein have confirmed that long-term protection with appropriate pesticides that reduce $A$. yasumatsui infestations enables $C$. micronesica trees to accumulate stem carbohydrates and generate $100 \%$ success in adventitious root formation on large stem cuttings. The direct relationship between carbohydrate status at the time of stem excision and propagation success rates indicates that the inclusion of a plant physiologist with knowledge of source-sink relations may be a prerequisite for success in C. micronesica rescue projects.

The choice to use field sites with native soils as the propagation substrate was considered for two reasons. First, Guam's frequent tropical cyclones snap C. micronesica stem sections, and before the invasions of specialist cycad pests, these fallen stems developed adventitious roots to enable an effective natural form of clonal propagation (Marler and Lawrence, 2013). The protocols in this manipulative study mirrored this natural cloning. Second, one of the most expensive components of rescue projects (Marler and Cruz, 2017) is the managed nursery phase. The knowledge that healthy C. micronesica stems can successfully form adventitious roots following insertion into native field soil enables the potential to

Table 1. Carbohydrate status of large Cycas micronesica stem cuttings as influenced by two levels of Aulacaspis yasumatsui protection by imidacloprid. Tissue was harvested $1 \mathrm{~m}$ from the stem apex and included homogenized periderm, cortex, vascular, and pith tissues. Means are samples combined from two locations, $n=20$.

\begin{tabular}{lccrr}
\hline Trait $\left(\mathrm{mg} \cdot \mathrm{g}^{-1}\right)$ & Protected, healthy & Unprotected, unhealthy & $F$-value & $P$-value \\
\hline Fructose & 14.25 & 5.95 & 30.12 & $<0.0001$ \\
Glucose & 23.75 & 5.40 & 89.23 & $<0.0001$ \\
Sucrose & 130.85 & 63.15 & 85.47 & $<0.0001$ \\
Maltose & 0.79 & 0.40 & 0.40 & 0.5334 \\
Starch & 198.90 & 121.80 & 126.35 & $<0.0001$ \\
Sugars & & 74.90 & 121.87 & $<0.0001$ \\
Carbohydrates $^{y}$ & 169.64 & 196.70 & 210.24 & $<0.0001$ \\
Sugars/starch $^{\text {s }}$ & 368.54 & 1.84 & 9.22 & 0.0071 \\
\hline
\end{tabular}

${ }^{\mathrm{z}}$ Sum of the four sugars.

${ }^{\mathrm{y}}$ Sum of all five carbohydrates.

HortScience Vol. 53(8) August 2018

bypass the expensive nursery phase during future tree rescue projects.

More refining of how $A$. yasumatsui infestations influence carbohydrates of host Cycas trees is warranted. One deficiency in our knowledge is how $A$. yasumatsui infestations influence source-sink strategies of Cycas trees to define three-dimensional carbohydrate status. The sampling protocol in this study and our previous study (Marler and Cascasan, 2018) homogenized all radial stem tissues, including periderm, cortex, vascular cylinders, and pith. Therefore, nothing is known to date about how the declines in stem carbohydrates following $A$. yasumatsui herbivory are influenced by radial or axial stratification within the host tree. This lack of systematic data obfuscates our ability to fully understand optimal allocation of nutrients and metabolites in Cycas plants under biotic stress.

The conservation of terrestrial tree species should progress with the input of all stakeholders and the implementation of information from all disciplines. This threatened tree species has been ESA-listed as a result of plant mortality associated with ubiquitous non-native insect herbivores. The empirical information herein is valuable for utilitarian purposes for more fully understanding asexual propagation of Cycas stems. However, the added knowledge does not address the primary threat that is endangering the tree species. As a result, large-scale use of stem cuttings to rescue $C$. micronesica trees may serve to successfully remove the specimens from the identified military construction sites, but these rescued trees will subsequently succumb to the ubiquitous biological threats shortly after the culmination of the conservation projects (Marler and Lindström, 2017). The accumulation of all relevant information to date indicates that human and budgetary resources should not be expended on expensive projects that will almost certainly be unsuccessful, but should instead be spent on developing a sustainable approach to mitigating the primary threat of $A$. yasumatsui infestations.

In summary, chronic $A$. yasumatsui herbivory of large $C$. micronesica trees caused declines in stem carbohydrates and limited success rates for asexual propagation by stem cuttings. The results add to the growing foundation of knowledge: 1) nonstructural carbohydrates of Cycas plants are intimately involved in the means by which $A$. yasumatsui threatens its host species, 2) the use of large stem cuttings to rescue $C$. micronesica trees from future construction sites is not recommended if historical A. yasumatsui infestations have not been controlled by insecticides, 3) considerable storage of carbohydrates in healthy $C$. micronesica stems enables successful asexual propagation even if no horticultural after-care is provided, 4) cycad conservation projects will reach the greatest level of success when the need for involvement of a sourcesink plant physiologist is honored, and 5) refinement of conservation decisions would benefit from developing a quick field test for 
practitioners that could serve as a proxy for actual carbohydrate quantification of Cycas trees.

\section{Literature Cited}

Cascasan, A.N. and T.E. Marler. 2016. Publishing trends for Cycadales, the most threatened plant group. J. Threat. Taxa 8:8575-8582.

Clegg, K.M. 1956. The application of the anthrone reagent to the estimation of starch in cereals. J. Sci. Food Agr. 7:40-44.

Cooke, S.J., A.M. Rous, L.A. Donaldson, J.J. Taylor, T. Rytwinski, K.A. Prior, K.E. Smokorowski, and J.R. Bennett. 2018. Evidence-based restoration in the Anthropocene-From acting with purpose to acting for impact. Restor. Ecol. 26:201-205.

Fisher, J.B., A.J. Lindström, and T.E. Marler. 2009. Tissue responses and solution movement after stem wounding in six Cycas species. HortScience 44:848-851.

Marler, T., J. Haynes, and A. Lindström. 2010. Cycas micronesica. The IUCN red list of threatened species. Version 2014.3. 30 May 2018. <www.iucnredlist.org>.

Marler, T.E. 2012. Cycad aulacaspis scale invades the Mariana Islands. Mem. N. Y. Bot. Gard. 106:20-35.
Marler, T.E. 2017. Horticultural research crucial for plant conservation and ecosystem restoration. HortScience 52:1648-1649.

Marler, T.E. and A.N.J. Cascasan. 2018. Carbohydrate depletion during lethal infestation of Aulacaspis yasumatsui on Cycas revoluta. Intl. J. Plant Sci. 179:497-504.

Marler, T.E. and G.N. Cruz. 2017. Adventitious rooting of mature Cycas micronesica K.D. Hill tree stems reveals moderate success for salvage of an endangered cycad. J. Threat. Taxa 9:10565-10570.

Marler, T.E. and N. Dongol. 2016. Seed ontogeny and nonstructural carbohydrates of Cycas micronesica megagametophyte tissue. HortScience 51:1144-1147.

Marler, T.E. and J.H. Lawrence. 2012. Demography of Cycas micronesica on Guam following introduction of the armoured scale Aulacaspis yasumatsui. J. Trop. Ecol. 28:233-242.

Marler, T.E. and J.H. Lawrence. 2013. Phytophagous insects reduce cycad resistance to tropical cyclone winds and impair storm recovery. HortScience 48:1224-1226.

Marler, T.E. and A.J. Lindström. 2014. The value of research to selling the conservation of threatened species: The case of Cycas micronesica. J. Threat. Taxa 6:6523-6528.

Marler, T.E. and A.J. Lindström. 2017. First, do no harm. Commun. Integr. Biol. 10:e1393593.
Marler, T.E. and R. Muniappan. 2006. Pests of Cycas micronesica leaf, stem, and male reproductive tissues with notes on current threat status. Micronesica 39:1-9.

Norstog, K.J. and T.J. Nicholls. 1997. The biology of the cycads. Cornell Univ. Press, New York, NY.

Pullin, A.S. and T.M. Knight. 2009. Doing more good than harm-Building an evidence-base for conservation and environment management. Biol. Conserv. 142:931-934.

Schloter, M., J.B. Winkler, M. Aneja, N. Koch, F. Fleischmann, K. Pritsch, W. Heller, S. Stich, T.E. Grams, A. Göttlein, R. Matyssek, and J.C. Munch. 2005. Short term effects of ozone on the plant-rhizosphere-bulk soil system of young beech trees. Plant Biol. (Stuttg) 7:728736.

United States Fish \& Wildlife Service. 2015. Endangered and threatened wildlife and plants: Endangered status for 16 species and threatened status for 7 species in Micronesia. Fed. Regist. 80:59424-59497.

Young, F.J. 1988. Soil survey of Territory of Guam. U.S. Dept. Agr. Soil Conservation Service.

Zedler, J.B. 2017. What's new in adaptive management and restoration of coasts and estuaries? Estuaries Coasts 40:1-21. 